\title{
Investigation on the printability of bioink based on alginate-gelatin hydrogel and liquid crystals
}

\author{
Alyaa Idrees Abdulmaged ${ }^{1}$, Chin Fhong Soon ${ }^{2}$, Balkis A. Talip ${ }^{3}$, \\ Sheril Amira Othman ${ }^{4}$, Gim Pao Lim ${ }^{5}$, Kian Sek Tee ${ }^{6}$ \\ ${ }^{1,3}$ Faculty of Applied Sciences and Technology, Universiti Tun Hussein Onn Malaysia Pagoh Campus, \\ Hub Pendidikan Tinggi Pagoh, Malaysia \\ ${ }^{2,5}$ Microelectronics and Nanotechnology-Shamsuddin Research Centre, Institute of Integrated Engineering, \\ University Tun Hussein Onn Malaysia, Malaysia \\ ${ }^{2,4,6}$ Faculty of Electrical and Electronic Engineering, University Tun Hussein Onn Malaysia, Malaysia
}

\begin{tabular}{l} 
Article Info \\
\hline Article history: \\
Received Dec 10, 2019 \\
Revised Feb 14, 2020 \\
Accepted Mar 23, 2020 \\
\hline Keywords: \\
Alginate \\
Extrusion-based 3D bioprinting \\
biomaterials \\
Gelatin \\
Liquid crystals \\
Tissue engineering
\end{tabular}

\section{Corresponding Author:}

Chin Fhong Soon,

Faculty of Electrical and Electronic Engineering,

University Tun Hussein Onn Malaysia, Parit Raja, 86400 Johor, Malaysia.

Email: soon@uthm.edu.my

\begin{abstract}
Bioinks of 3D bioprinting have significant potential application in the field of tissue engineering to support cell attachment and proliferation. In this work, the alginate-gelatin-CELC (AGLC) bioink based on different compositions of alginate-gelatin (AG) hydrogel and cholesteryl ester liquid crystals (CELC) was prepared. Primarily, the alginate-gelatin hydrogel with certain concentration of gelatin $(10-50 \% \mathrm{w} / \mathrm{v})$ was investigated. The printability of the hydrogel reached a minimum width of $1.8 \mathrm{~mm}$ at a flow rate of $1 \mathrm{~mL} / \mathrm{min}$ when the gelatin concentration was increased to $50 \% \mathrm{w} / \mathrm{v}$ (AG1050). Subsequently, the respective polymers with $10 \% \mathrm{w} / \mathrm{v}$ alginate and $50 \% \mathrm{w} / \mathrm{v}$ gelatin blended with $1 \%, 5 \%, 10 \%, 20 \%, 40 \%$, and $60 \% \mathrm{w} / \mathrm{v}$ of CELC in the preparation of the alginate-gelatin-CELC bioink was further investigated. The printability of the bioink was examined by micro-extrusion based 3D bioprinter. The printability of the bioink enhanced by $27.8 \%$ as compared to AG1050 and reached a minimum width of $1.3 \mathrm{~mm}$ at a flow rate of $1 \mathrm{~mL} / \mathrm{min}$ when the CELC concentration was increased to $40 \%$ and $60 \%$. The tested properties of the bioink show that the CELC improve shear-thinning and lipid moieties properties to the composite bioink and hence, enhances its printability.
\end{abstract}

This is an open access article under the CC BY-SA license.

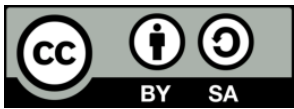

\section{INTRODUCTION}

In the past few decades, three-dimensional printing was developed with the aid of computer-aided design (CAD) and its application was extended to the field of biomedical engineering and applications [1,2]. The recent development of biocompatible materials for 3D bioprinting systems enable tissue engineering research, so as to develop complex tissue-like structure which can be used for restoring, replacing or regenerating damaged tissues and organs [3]. The 3D bioprinting technology is a process of integrating and accurately depositing biomaterials, living cells and growth factors in a layer by layer arrangement $[4,5]$. It entails a bioink that is compatible to a specific extent with the biological system and achieves high-quality printing results [6]. Printability is an important property of 3D bioprinting process as the produced bioink for this process must manifest for both the cellular architecture and shape fidelity. A successful printing of hydrogel relies on achieving high resolution and maintains the high integrity of the printed structures [7]. 
The development of a bioink for 3D bioprinting is challenging due to simultaneous optimization of high-resolution printing, preserving mechanical structures and biocompatibility [8]. The aim is to achieve high resolution and shape fidelity post printing. Different studies attempted to develop a multi-materials bioink for 3D cell culture to create in-vitro 3D tissue-like constructs with different moieties. Different materials from both natural and synthetic sources have been utilized in the fabrication and development of bioinks. These materials include alginate, gelatin, chitosan, agarose, cellulose, polylactic acid, and poly-ethylene-glycol [9]. Alginate is a natural material used in bioink fabrication due to its shear-thinning properties and structural similarity to the extra cellular matrix (ECM) of native tissue [10]. However, fabricating a bioink from pure alginate hydrogel is challenging due to the low viscosity of alginate solution, in which the dispensed filaments can be easily degraded and collapsed, hence, a well-defined shape of the 3D bioprinted structures is difficult to achieve $[11,12]$. Subsequently, alginate-gelatin hydrogel has been widely investigated in the 3D bioprinting due to their biocompatibility and support for cell viability. To overcome this limitation, different means have been implemented to improve the printability of alginate hydrogels, which including crosslinking with ionic solutions, tuning of printing parameter and blending with other polymers [6, 12]. Further, blending of alginate with other polymers such as gelatin [13], chitosan [12], and cellulose nanocrystals (CNCs) [14] is an effective strategy to improve the printability of alginate-based bioinks.

Giuseppe et al. [15] investigated the printability, mechanical properties and cell viability of alginate-gelatin hydrogel by varying each constituent concentration. Their results demonstrated good printability and mechanical properties of alginate-gelatin composites hydrogel with an increase in the viscosity of the hydrogel. Chung et al. [6] studied the rheological properties of alginate and alginate-gelatin hydrogel. They indicated that the ink solution made with pure alginate behaves like a liquid with insufficient stiffness to hold the shape of the printed structure. While alginate-gelatin showed more gel-like behavior and better resolution for the printed scaffolds. This indicates that gelatin increased the initial viscosity and mechanical stability of hydrogel ink to be printable.

Liquid crystal (LC) is a new class of biomaterials that found great interest and ascending attention from the scientific community for its use as smart advanced materials in scaffolds engineering [16]. In this context, Du et al. [17] designed a biomimetic composite hydrogel that consists of chitosan and hydroxypropyl cellulose ester liquid crystals (CS/LCs). Cell viability test and cell morphology observation for cultured 3T3 fibroblast cells in CS/LCs composite exhibited high viability, spherical shape and the cells thrived and secreted its own ECM on CS/LC composite surface within 7 days of cultivation. The embedded molecules of LCs in the soft matrix of chitosan and the optimum hydrophilic-hydrophobic balance of CS/LC composite provide favorable interface adhesive domains for cells attachment and proliferation. Soon et al. [18] demonstrated the culturing of human-derived keratinocytes (HaCaT) on the cholesteryl ester liquid crystals (CELC) coated substrate resulted in secretion of their own extracellular matrix proteins. In addition, the presence of cholesterol and lipid ester moieties in the chemical structure of the CELC increased the lipid moieties in the culture and thus, creating an adhesion layer preferred by the cells adhesion in the biological system. Cholesterol is essential for cell membrane formation and stabilization [18, 19]. The lyotropic phase and cholesteryl ester compound of the liquid crystals mimic the in-vivo system may be the factors attracting the affinity of cells.

This research aims to develop a new bioink that simulate the natural ECM of native tissues. The main problem that this paper attempted to solve is to further improve the printing resolution and shape integrity of the alginate-gelatin (AG) 3D printed structure. Liquid crystal made a potential solution due to its shear-thinning properties that promotes cell attachment and proliferation. Subsequently, in this paper, the influence of gelatin on the printability of AG hydrogel was initially explored in which, alginate was blended with different ratio of gelatin and liquid crystals. A wide range of CELC concentrations (1\% to $60 \%$ ) was introduced to AG hydrogel to increase the lipid moieties in the bioink and for studying the effects of CELC concentrations on AG hydrogel printability. Meanwhile, the proposed solution is a new alginate-gelatin-CELC (AGLC) bioink that combines the AG hydrogel with the CELC. The developed alginate-gelatin-CELC (AGLC) bioink of this work showed high printability according to the tissue engineering standards of 3D bioprinting.

\section{RESEARCH METHOD}

\subsection{Materials}

Sodium alginate $(0.01 \mathrm{~g} / \mathrm{mL}$, viscosity, guluronic to manuronic ratio $39 \%$ to $61 \%)$, cholesteryl oleyl carbonate $\left(\mathrm{C}_{46} \mathrm{H}_{80} \mathrm{O}\right)$, cholesteryl perlagonate $\left(\mathrm{C}_{36} \mathrm{H}_{62} \mathrm{O}_{2}\right)$, and cholesteryl chloride $\left(\mathrm{C}_{27} \mathrm{H}_{45} \mathrm{Cl}\right)$ and gelatin powder used in this work to fabricate alginate-gelatin (AG) and alginate-gelatin-cholesteryl ester liquid crystals (AGLC) bioinks. 


\subsection{Preparation of alginate-gelatin hydrogel}

Table 1 shows the AG hydrogels synthesized using different concentrations of gelatin. 10\% w/v of alginate solution was prepared by dissolving $1 \mathrm{~g}$ of sodium alginate powder into $10 \mathrm{~mL}$ of de-ionized water at room temperature. Different concentrations of gelatin (as shown in Table 1) solutions were prepared by dissolving gelatin powder into $10 \mathrm{~mL}$ of de-ionized water at $60^{\circ} \mathrm{C}$. The gelatin solution was constantly stirred using a magnetic stirrer on a hot plate at a speed of $750 \mathrm{rpm}$. All hydrogel solutions were centrifuged for $1 \mathrm{~min}$ at $1200 \mathrm{rpm}$ to remove air bubbles.

Table 1. AG hydrogel

\begin{tabular}{cc}
\hline Sample & Alginate: Gelatin (w/v : w/v \%) \\
\hline AG1010 & $10 \%$ Alginate: $10 \%$ Gelatin \\
AG1020 & 10\%Alginate:20\%Gelatin \\
AG1030 & 10\%Alginate:30\%Gelatin \\
AG1040 & $10 \%$ Alginate:40\%Gelatin \\
AG1050 & 10\%Alginate:50\%Gelatin \\
\hline
\end{tabular}

\subsection{Preparation of alginate-gelatin-CELC bioink}

CELC biomaterial sample used in this work were formulated using $37.5 \%$ of cholesteryl oleyl carbonate $37.5 \%$ of cholesteryl Perlagonate and $25.0 \%$ of cholesteryl chloride, and prepared in a $50 \mathrm{~mL}$ glass vial, the solid mixtures of the three liquid crystals were heated on a hot plate to their isotropic phase at the temperature ranged between $115-120^{\circ} \mathrm{C}$ [20]. To prepare AGLC bioink, different volumes of prepared CELCs biomaterial sample (isotropic phase) added to AG1050 solution and the blend stirred manually to obtain homogeneous solutions denoted as AGLC105001, AGLC105005, AGLC105010, AGLC105020, AGLC105040, and AGLC105060. Table 2 shows the AGLC bioink prepared with different concentration of CELC.

Table 2. The concentrations of AG1050-CELC bioink

\begin{tabular}{ccl}
\hline No. & Sample name & Alginate-gelatin-CELC \\
\hline 1 & AGLC105001 & AG1050-1\% \\
2 & AGLC105005 & AG1050-5\% \\
3 & AGLC105010 & AG1050-10\% \\
4 & AGLC105020 & AG1050-20\% \\
5 & AGLC105040 & AG1050-40\% \\
6 & AGLC105060 & AG1050-60\% \\
\hline
\end{tabular}

\subsection{Evaluation of printability}

Printability of the AG hydrogel and AGLC and their respective polymers were conducted using a micro-extrusion based 3D bioprinting system. Table 3 shows the experimental parameters of printability test of AG hydrogel and AGLC bioink. Different formulations of bioink were printed on the surface of the petri dish at room temperature $\left(25^{\circ} \mathrm{C}\right)$. All these operations were performed in a sterilized environment, on the clean bench. The results represent the average of three consecutive runs expressed in mean \pm standard deviation. An in-house micro-extrusion based 3D bioprinting system was used for the bioprinting. The bioprinting system consists of an extruder microcontroller, extruder machine and a 3D printer. Figure 1 shows the block diagram of the 3D micro-extrusion bioprinting system used for the bioprinting.

Table 3. The 3D bioprinting parameters

\begin{tabular}{clc}
\hline No. & \multicolumn{1}{c}{ Parameter } & Value \\
\hline 1. & Flow rate & $1-5 \mathrm{~mL} / \mathrm{min}$ \\
2. & Printing speed & $30 \mathrm{~mm} / \mathrm{s}$ \\
3. & Nozzle inner diameter & $1 \mathrm{~mm}$ \\
\hline
\end{tabular}

The extrusion settings of Figure 1 allow adjustment of the flow rates values from 1 to $5 \mathrm{~mL} / \mathrm{min}$ under diffusion and infusion modes. The 3D printer was programmed by G-code, numerical control programming language, to generate specific printed patterns of a square. The G-code directs the printing head to create the square shape using a series of G, X, Y, Z commands. A segment of the used G-code to print a square with a width of $3.5 \mathrm{~cm} \times 3.5 \mathrm{~cm} \times 3.5 \mathrm{~cm}$ is as shown in Figure 2. The G00, G01, and G21 are command that controls the movement of the printer head. G00 is the rapid travel command, G01 activates linear motion 
command, G20, and G21 commands instruct the controls of the movement in the unit of inch and $\mathrm{mm}$, respectively.

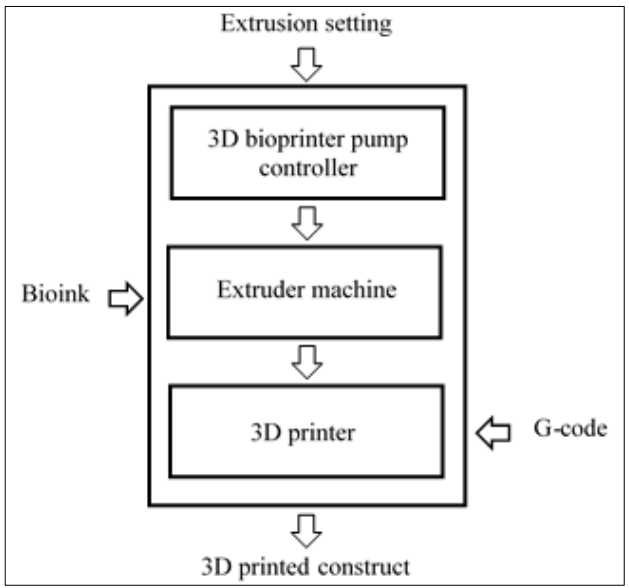

Figure 1. The block diagram of the 3D micro-extrusion bioprinting system design

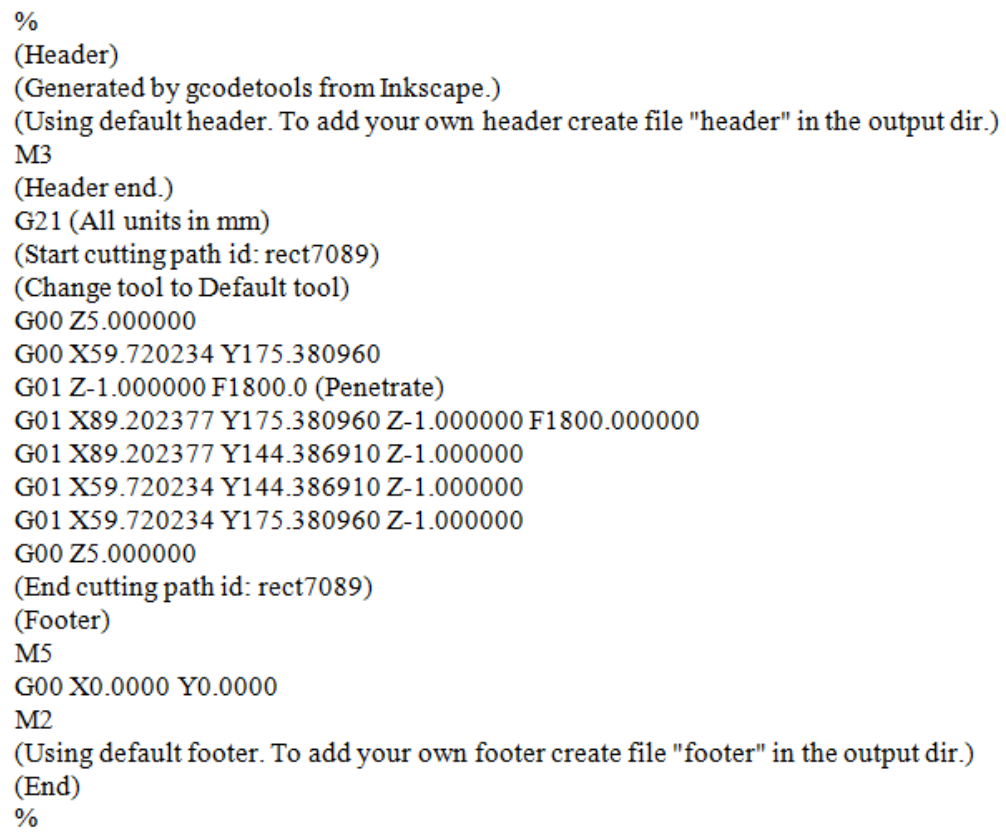

Figure 2. The G-code customized for the 3D printer

\section{RESULTS AND DISCUSSION}

\subsection{The effects of gelatin concentration on the printability of alginate-gelatin bioink}

The images of Figures 3 and 4 show the printability of the AG hydrogel at different concentrations of gelatin and at a fixed flow rate of $1 \mathrm{~mL} / \mathrm{min}$. Figure 3 shows that the designed pattern could not be printed at a low concentration of gelatin $(10 \% \mathrm{w} / \mathrm{v})$. Similar results have been shown for the second test with a concentration of $20 \% \mathrm{w} / \mathrm{v}$ gelatin. It was observed that AG hydrogel with low gelatin concentrations $(10 \%, 20 \%$, and $30 \% \mathrm{w} / \mathrm{v})$ exhibited a fluid-like behavior with poor printability of the designed pattern. This fluidic behavior of alginate is as suggested by a previous report in [6]. The AG1040 and AG1050 hydrogels resulted in a well-defined lining and small width of printed filaments. Among the five different concentrations, AG1050 provided the thinnest printed filament line width at $1.8 \mathrm{~mm}$ and the AG1020 provided a wider printed filament line width of $3 \mathrm{~mm}$ on average. The filaments with large width of printed 
structures produced are associated with fluid-like behavior of bioink inferred that low viscosity and stiffness of the solution affected the post-printing shape fidelity [7]. With an increase in the concentration of gelatin up to $40 \% \mathrm{w} / \mathrm{v}$, the filament width decreased, and the resolution of the printed pattern improved with a filament width of $2.0 \mathrm{~mm}$ when the flow rate was set at $1 \mathrm{~mL} / \mathrm{min}$. The printability was found to be level off and reached a minimum width of $1.8 \mathrm{~mm}$ when the gelatin concentration was increased to $50 \% \mathrm{w} / \mathrm{v}$ at a flow rate of $1 \mathrm{~mL} / \mathrm{min}$. However, when the concentration of gelatin was above $50 \% \mathrm{w} / \mathrm{v}$, the AG hydrogel was difficult to be extruded through the nozzle. This was due to the high viscosity forming high resistance for hydrogel to flow. Obviously, a concentration of gelatin up to $50 \% \mathrm{w} / \mathrm{v}$ has improved the printability of the bioink.

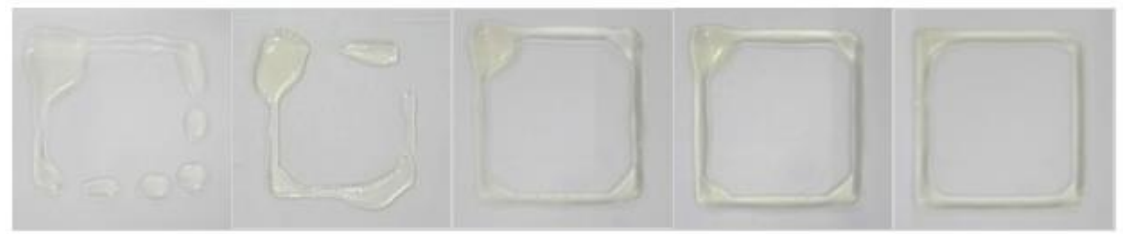
(a) $\mathrm{AG} 1010$
(b) AG1020
(c) AG1030
(d) AG1040
(e) AG1050

Figure 3. The printed constructs produced at different concentrations of the AG hydrogel at a fixed flow rate of $1 \mathrm{~mL} / \mathrm{min}$

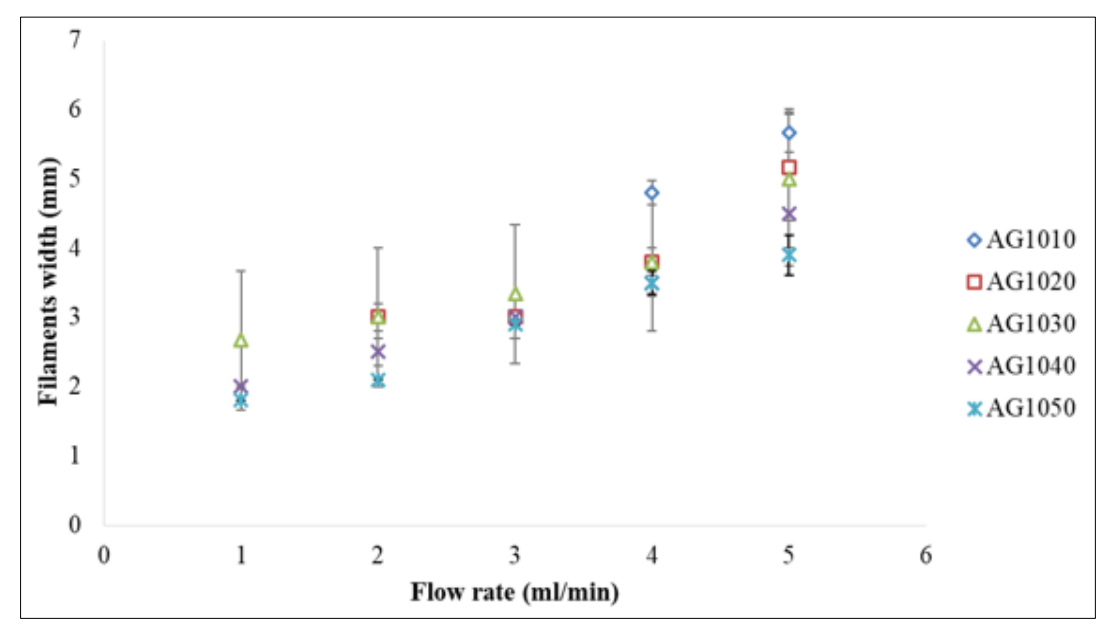

Figure 4. The relationship of filament width to the flow rate of different concentrations of AG bioink

Figure 4 shows the results of filament width influenced by the different flow rate and concentration of AG bioink. The AG1010 hydrogel at 1 to $3 \mathrm{~mL} / \mathrm{min}$ flow rates exhibited an incomplete pattern of the printed filament for measurement (as shown in Figure 3). Subsequently, the filament width of AG1010 hydrogel at the flow rates of 4 and $5 \mathrm{~mL} / \mathrm{min}$ showed a high extent of spreading. The AG1020 hydrogel (with a concentration of $20 \% \mathrm{w} / \mathrm{v}$ gelatin) at $1 \mathrm{~mL} / \mathrm{min}$ flow rate also exhibited an incomplete pattern of the printed filament. While the flow rate of 2 to $5 \mathrm{~mL} / \mathrm{min}$ increased the filament width of the hydrogel from 3.0 to $5.5 \mathrm{~mm}$ as shown in Figure 4. Next, the AG1030 hydrogel (with the concentration of $30 \% \mathrm{w} / \mathrm{v}$ gelatin) exhibited better results but the width of the printed filaments linearly increased from 2.5 to $3.8 \mathrm{~mm}$ with an increase of the flow rates from 1 to $4 \mathrm{~mL} / \mathrm{min}$. The width of the filament increased to $5.0 \mathrm{~mm}$ when the flow rate reached $5 \mathrm{~mL} / \mathrm{min}$. Hence, the AG1010, AG1020 and AG1030 showed poor printability due to the low viscosity of the formulated alginate hydrogel with $10 \%-30 \% \mathrm{w} / \mathrm{v}$ of gelatin $[21,22]$.

The AG1040 hydrogel with $40 \%$ w/v gelatin concentration demonstrated a thinner printed filaments width of $2.0 \mathrm{~mm}$ compared with the previously examined hydrogels at $1 \mathrm{~mL} / \mathrm{min}$ of flow rate. AG1050 showed higher printing resolution and thinner filaments width of $1.8 \mathrm{~mm}$. Hence, the results showed that an increase in the concentration of the gelatin increased the viscosity of the hydrogel which offered higher printing consistency and better control of printing width. However, an increase in the concentration of the gelatin above $50 \% \mathrm{w} / \mathrm{v}$ produced highly viscous bioink and the bioink was difficult to be extruded. 
Additionally, the increase of the flow rate from $1-5 \mathrm{~mL} / \mathrm{min}$ generally increased the width of the printed filaments in all the tested hydrogels. These results agreed with the previous studies reported by Chung et al. [6] and Giuseppe et al. [15].

The key requirements of successful bioprinting bioink for micro-extrusion-based 3D bioprinting include high printing resolution (thin filaments), proper mechanical properties (viscosity, stiffness) to support the printed structures and well-defined shape after extrusion [23]. These properties were better demonstrated by the hydrogel formulated with $10 \%$ alginate and 50\% gelatin (AG1050) compared with the other tested mixtures. Therefore, in the present research, AG1050 was selected for further experiments with CELC to investigate the effects of CELC in different concentrations on the printability of alginate-gelatin-LC bioink.

\subsection{The effects of CELC concentration on the printability of alginate-gelatin-LC bioink}

Figure 5 shows the results of the printability of alginate-gelatin-LC bioink in different compositions in square patterns denoted asAGLC105001, AGLC105005, AGLC105010, AGLC105020, AGLC105040, and AGLC105060. It was observed that the bioink prepared with additions of different CELC concentrations show improved printability in comparison with similar print parameters for printing AG. CELC is very much functioning as a lubricant to the AG hydrogels enabling printing high fidelity constructs.

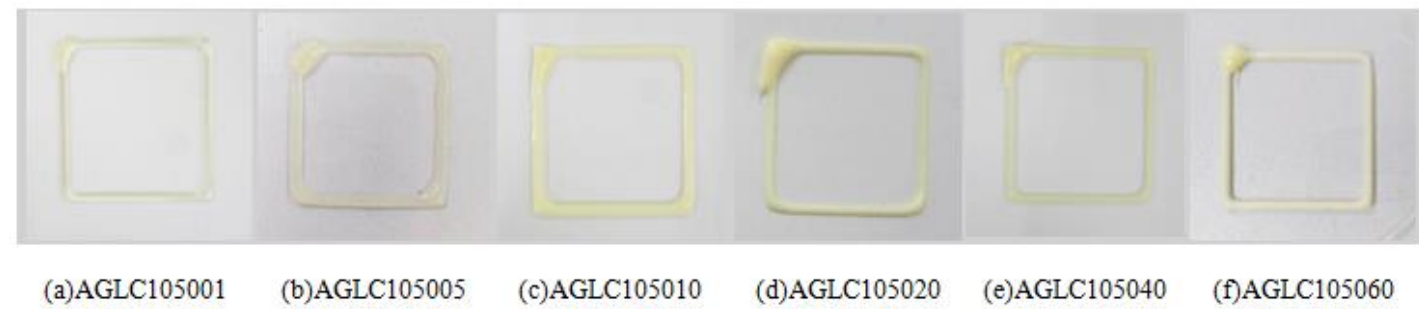

Figure 5. The printed constructs produced at different concentrations at, (a) $1 \%$, (b) $5 \%$, (c) $10 \%$, (d) $20 \%$, (e) $40 \%$, and (f) $60 \%$ of AGLC bioink at a fixed flow rate of $1 \mathrm{~mL} / \mathrm{min}$

Furthermore, the results obtained from AGLC hydrogel and their respective polymer at different flow rates $(1-5 \mathrm{~mL} / \mathrm{min})$ are as shown in the Figure 6. Based on Figure 6, it can be observed that an increase in the concentration of CELC results in more accurate printing and thinner filament width of the printed pattern. However, bioink formulated with $1 \%$ of CELC did not show noticeable change on the filaments width compared with the AG1050 hydrogel. Increasing the concentration of CELC to 5\% resulted in a decrease in the width of printed filaments, with a minimum width of $1.6 \mathrm{~mm}$ obtained when the flow rate was set at $1 \mathrm{~mL} / \mathrm{min}$. Further increases in CELC concentration to 10\% (AGLC105010) shows a slight decrease in the width of printed filaments (as shown in Figure 6). The minimum width of printed filaments achieved in this concentration was $1.5 \mathrm{~mm}$ at the flow rate of $1 \mathrm{~mL} / \mathrm{min}$. The bioink which was formulated with $20 \%$ of CELC (AGLC105020) showed similar results to the bioink with the $10 \%$ of CELC (AGLC105010) in which, no significant differences in the width of the printed filaments. It was indicated with the minimum filament width of $1.5 \mathrm{~mm}$ at the flow rate of $1 \mathrm{~mL} / \mathrm{min}$. The bioink that was synthesized with $40 \%$ of CELC (AGLC105040) resulted in minor effects on the width of the printed filaments with minimum filaments up to $1.3 \mathrm{~mm}$ at the flow rate of $1 \mathrm{~mL} / \mathrm{min}$. The bioink composed with $60 \%$ of CELC (AGLC105060) exhibited the same effect of the bioink with $40 \%$ of CELC on the width of printed filaments. It has the same minimum filaments width of $1.3 \mathrm{~mm}$ at the flow rate $1 \mathrm{~mL} / \mathrm{min}$.

In addition, increasing the flow rate more than $1 \mathrm{~mL} / \mathrm{min}$ for all AGLC bioinks resulted in an increasein the width of the printed filaments which in turn, affected the resolution of the printed structures. Further increase in CELC concentration above $60 \%$ produced highly viscous and unprintable hydrogel in which it cannot be extruded by the 3D bioprinter. However, it was also observed that the standard deviation of the width of the printed filament decreased when the concentration of the CELC based bioink increased. Hence a more viscous hydrogel offers higher printing consistency and better control over the printed filament widths. The print fidelity and high resolution of the printed structures improved through an increase in CELC concentration as observed from the sharp corners of the printed square structure.

The blends of AGLC105040 and AGLC105060 produced the thinnest filament width of $1.3 \mathrm{~mm}$. Hence, these two bioinks manifest high resolution and shape fidelity post-printing. The addition of LC has improved the printability and shape fidelity of the 3D printing [24]. The reason for this improvement is that the viscosity of the hydrogel increased with the increase of the CELC concentration which resulted in a decrease in the width of the filaments. Additionally, the shear-thinning properties of the CELC facilitate lubricate 
the extrusion of AGLC bioink through the nozzle. A previous study by Soon et al. [20] on the rheological behavior of CELC provided good details about the viscoelastic nature of CELC. Based on this study, the shear-thinning behavior of CELC was observed when the complex viscosity decreased with the increased in shear rate, illustrating another desirable characteristic of CELC for extrusion-based bioprinting.

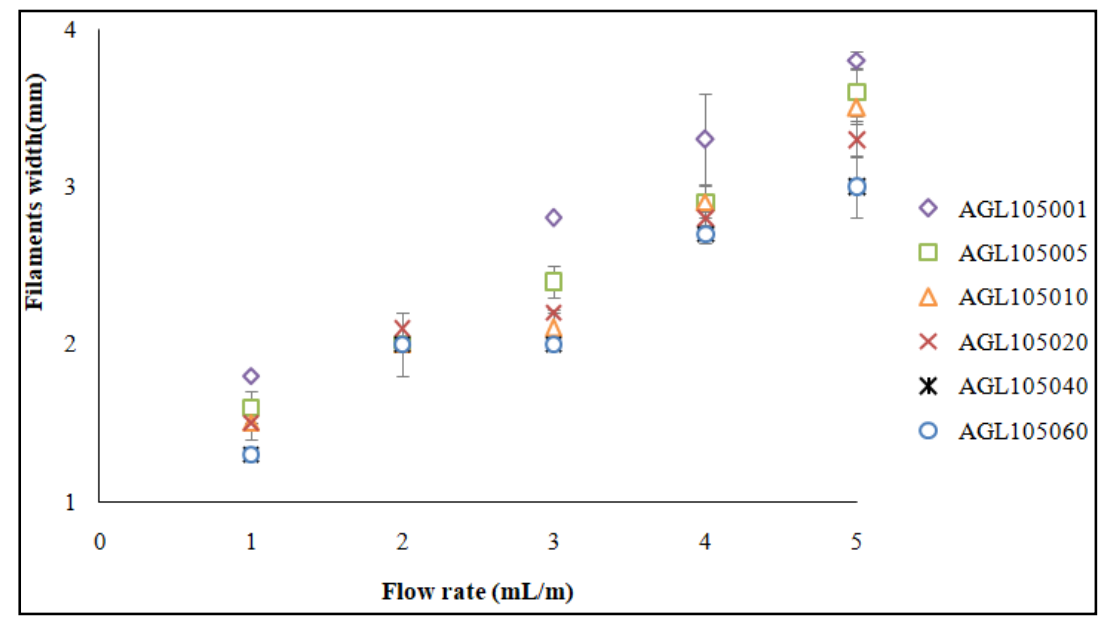

Figure 6. The relationship of filaments width to the flow rate of different concentration of AGLC bioink

Subsequently, the non-Newtonian behavior of AGLC bioink caused a decrease in viscosity when shear stress was applied which allowed the AGLC bioink to flow like a liquid through the nozzle during the printing process, and upon the deposition, the shear stress decreased causing a rapid recovery in the viscosity to its original state, which explained the flow of a viscous bioink formulated with $20 \%, 40 \%$, and $60 \%$ of CELC (AGLC105020, AGLC105040, and AGLC105060) from printing head. Hence, shear-thinning bioink that continuously flow when subjected to shear stress, but a rapid reassembly of the network to a hydrogel once the shear was removed, are preferable materials for use in the micro-extrusion based bioprinting [24, 25]. Shear-thinning, high resolution and shape integrity of printed structures produced using AGLC105040 and AGLC105060 as suitable hydrogel inks for 3D bioprinting.

\section{CONCLUSION}

In this work, the printability of an alginate-gelatin-CELC bioink was investigated by varying the concentration of gelatin, cholesteryl ester liquid crystals (CELC) and flow rate. Printability means an accurate and precise deposition of printed filaments. The printability of alginate-gelatin hydrogel can be further increased via the addition of the liquid crystal due to its shear-thinning properties. Whilst containing lipid moiety, the liquid crystal may have the biochemistry property to promote cell attachment and proliferation. Subsequently, the AGLC bioink was prepared and printed successfully using 3D micro-extrusion bioprinting technique. The results showed that concentration of gelatin at $50 \% \mathrm{w} / \mathrm{v}$ and concentration of CELC at $40 \%$ $\mathrm{w} / \mathrm{v}$ and $60 \% \mathrm{w} / \mathrm{v}$ extruded data flow rate of $1 \mathrm{~mL} / \mathrm{min}$, provided suitable printability. Shear thinning is an important property of CELC which facilitated the extrusion of the AGLC bioink and achieved high resolution of the structures post-printing. The produced AGLC bioink and its respective polymers may serve as a potentially multi-materials bioink for 3D bioprinting to create in-vitro $3 \mathrm{D}$ tissue-like constructs with different moieties.

\section{ACKNOWLEDGEMENTS}

This project is funded by the Ministry of Education Malaysia under Fundamental Research Grant Scheme (FRGS) with a reference number of Ref: FRGS/1/2018/STG05/UTHM/02/3. The authors also acknowledge support from Faezahana Mohkter and Ahmad Nasrull Mohamed for their technical assistance. 


\section{REFERENCES}

[1] Md. S. Islam, M. I. Ibrahimy, S. M. A. Motakkaber, A. K. M. Z. Hossain, and S. M. K. Azam, "Microstrip patch antenna with defected ground structure for biomedical application," Bulletin of Electrical Engineering and Informatics, vol. 8, no. 2, pp. 586-595, 2019.

[2] W. M. E. A. W. Jusoh and S. H. Ruslan, "Design and analysis of current mirror OTA in $45 \mathrm{~nm}$ and $90 \mathrm{~nm}$ CMOS technology for bio-medical application," Bulletin of Electrical Engineering and Informatics, vol. 9, no. 1, pp. 86-95, 2020.

[3] E. S. Bishop et al., "3-D bioprinting technologies in tissue engineering and regenerative medicine: Current and future trends," Genes \& Diseases, vol. 4, no. 4, pp. 185-195, 2017.

[4] I. T. Ozbolat, "Application areas of 3D bioprinting," Drug Discovery Today, vol. 21, no. 8, pp. 1257-1271, 2016.

[5] L. Ning, et al., "Bioprinting Schwann cell-laden scaffolds from low-viscosity hydrogel compositions," Journal of Materials Chemistry B., vol. 7, no. 29, pp. 4538-4551, 2019.

[6] J. H. Chung, et al., "Bio-ink properties and printability for extrusion printing living cells," Biomaterials Science, vol. 1, no. 7, pp. 763-773, 2013.

[7] S. Kyle, Z. M. Jeesop, A. Al-Sabah, and I. S. Whitaker, "Printability of candidate biomaterials for extrusion based 3D printing: State-of-the-art," Advanced Healthcare Materials, vol. 6, no. 16, pp. 1700-264, 2017.

[8] A. Panwar and L. Tan, "Current status of bioinks for micro-extrusion-based 3D bioprinting," Molecules, vol. 21, no. 6, pp. 1-26, 2016.

[9] A. Skardal and A. Atala, "Biomaterials for integration with 3-D bioprinting," Annals of Biomedical Engineering, vol. 43, no. 3, pp. 730-746, 2015.

[10] K. Y. Lee and D. J. Mooney, "Alginate: Properties and biomedical applications," Progress in Polymer Science, vol. 37, no. 1, pp. 106-126, 2012.

[11] M. H. Kim, Y. W. Lee, W-K. Jung, J. Oh and S. Y. Nam, "Enhanced rheological behaviors of alginate hydrogels with carrageenan for extrusion-based bioprinting," J. of the Mechan. Behav. of Biomed. Mat., vol. 9, pp. 187-194, 2019.

[12] Q. Liu, Q. Li, S. Xu, Q. Zheng, and X. Cao, "Preparation and properties of 3D printed alginate-chitosan polyion complex hydrogels for tissue engineering," Polymers, vol. 10, no. 6, pp. 1-13, 2018.

[13] B. Duan, L. A. Hockaday, K. H. Kang, and J. T. Butcher, "3D bioprinting of heterogeneous aortic valve conduits with alginate/gelatin hydrogels," J. of Biomedical Materials Research Part A , vol. 101, no. 5, pp 1255-1264, 2013.

[14] Y. Wu, Z. Y. W. Lin, A. C. Wenger, K. C. Tan, and X. S. Tang,"3D bioprinting of liver-mimetic construct with alginate/cellulose nanocrystal hybrid bioink," Bioprinting, vol. 9, pp. 1-6, 2018.

[15] M. Di Giuseppe et al., "Mechanical behaviorof Alginate-Gelatin hydrogels for 3D bioprinting," Journal of the Mechanical Behavior of Biomedical Materials, vol. 79, pp. 150-157, 2018.

[16] M. E. Prévôt, S. Ustunel, and E. Hegmann, "Liquid crystal elastomers-a path to biocompatible and biodegradable 3D-LCE scaffolds for tissue regeneration," Materials, vol. 11, no. 3, pp. 1-21, 2018.

[17] L. Du et al.,"Construction of physical crosslink-based chitosan/liquid crystal composite hydrogel and evaluation on their cytocompatibility," Regenerative Biomaterials, vol. 4, no. 1, pp. 39-45, 2016.

[18] C. F.Soon, et al.,"Biophysical characteristics of cells cultured on cholesteryl ester liquid crystals," Micron, vol. 56, pp. 73-79, 2014

[19] M. Mitov, "Cholesteric liquid crystals in living matter," Soft Matter, vol. 13, no. 23, pp. 4176-4209, 2017.

[20] C. F. Soon, M. Youseffi, T. Cough, N. Blagden, and M. C. T. Denyer, "Rheological characterization and in-situ investigation of the time-dependent cholesteric based lyotropic liquid crystals," Materials Science and Engineering: C, vol. 31, no. 7, pp. 1389-1397, 2011.

[21] T. Gao et al., "Optimization of gelatin-alginate composite bioink printability using rheological parameters: A systematic approach," Biofabrication, vol. 10, no. 3, pp. 1-27, 2018.

[22] T. Jiang et al., "Engineering bioprintable alginate/gelatin composite hydrogels with tunable mechanical and cell adhesive properties to modulate tumor spheroid growth kinetics," Biofabrication, vol. 12, no. 1, 2019.

[23] S. Adepu et al., "Three-dimensional bioprinting for bone tissue regeneration," Current Opinion in Biomedical Engineering, vol. 2, pp. 22-28, 2017.

[24] R. D. Murphy, S. Kimmins, A. J. Hibbitts, and A. Heise, "3D-extrusion printing of stable constructs composed of photoresponsive polypeptide hydrogels," Polymer Chemistry, vol. 10, no. 34, pp. 4675-4682, 2019.

[25] M. Hospodiuk, K. K. Moncal, M. Dey, and I. T. Ozbolat, "3D printing and biofabrication: Extrusion-based biofabrication in tissue engineering and regenerative medicine," References Series in Biomedical Engineering, Springer, pp. 255-281, 2016. 